\title{
Cities and Health: A Response to the Recent Commentaries
}

\author{
Michael K. Gusmano ${ }^{1}$, Victor G Rodwin ${ }^{2 *}$, Daniel Weisz ${ }^{3}$
}

*Correspondence to: Victor G Rodwin, Email: victor.rodwin@nyu.edu Copyright: $\odot 2015$ by Kerman University of Medical Sciences

Citation: Gusmano MK, Rodwin VG, Weisz D. Cities and health: a response to the recent commentaries. Int J Health Policy Manag. 2015;4(10):709-710. doi:10.15171/ijhpm.2015.149

Received: 27 July 2015, Accepted: 6 August 2015, ePublished: 8 August 2015

\section{$\mathrm{W}$} e are grateful to our many colleagues who took the time to respond to our analysis of Shanghai's declining "avoidable mortality." 1 The range of their perspectives across 5 recent commentaries reassures us that the topic is worthy of sustained study. Indeed, the presumption behind our comparative research on healthcare in world cities ${ }^{2}$ is that the city is a strategic unit of analysis for understanding the health sector and that world cities share a host of important characteristics. Contrary to Cheng's ${ }^{3}$ comment that we compared "disparate cities whose only common characteristic is that they are of mega-size," we have relied on a "most similar systems" approach to comparative analysis. ${ }^{4}$ World cities are characterized by high population size and density, similar commuting patterns between their outer rings and urban cores, and similar functions in the realms of international finance, culture, media, and provision of tertiary and quaternary medical care. Likewise, they exhibit flagrant socioeconomic inequalities, share many of the same strengths and weaknesses, but exist within nations with strikingly different health policies. Thus, comparisons among these cities, as well as within them (across neighborhoods), can shed light on the implications of national health policy for access to care, as well as on the importance of urban health policies for grappling with local health challenges.

We agree with Ren's comment ${ }^{5}$ that it would have been preferable to compare our data from New York City, Paris (and first ring) and Greater London to Shanghai City with a population of 10 million. Indeed, we attempted to do so, but the Shanghai Municipal Center for Disease Control and Prevention (SCDC) provided disaggregated mortality data, by cause, only for all of Shanghai. This problem reflects a broader issue in comparative urban health services research. While international comparisons are typically conducted across nation states with data assembled by leading international organizations (UN, OECD, WHO), there is no international association of large cities that provides comparable data for urban areas, their central cores, outer rings, let alone neighborhoods within them. This problem is well illustrated in the commentary by Yip and $\mathrm{Chen}^{6}$ where they present 2 graphs comparing life expectancy at birth across 6 cities. London, New York City and Hong Kong are comparable in terms of population size, but once again Shanghai probably refers to the whole city, Tokyo probably reflects the entire Prefecture that extends way beyond the most heavily urbanized areas, and Paris (if they did not include the first ring) includes only the 2.1 million population living in the dense and historic urban core.

The problem of inadequate data at the city-level is also the reason why Yip and Chen's ${ }^{6}$ suggestion that we assess healthadjusted life expectancy (HALE) and disability adjusted life years (DALY) for Shanghai is not practical. Putting aside the considerable ethical problems raised by how to weight different states of health, even in New York City for which we have a great deal of information about population health at the local level, we do not have sufficient data on prevalence of disease necessary to calculate such measures. Surely, as the world becomes increasingly urban, the demand for databases across world cities, as well as smaller ones, will increase and make it possible to deepen the kinds of research we began more than a decade ago. ${ }^{7,8}$

There is a final issue provoked by the indicator on which we based our analysis - amenable mortality (AM). We propose to address this issue in more detail because three of the commentaries raised it in different ways. Yan and Zhang' note the "unavoidable limitations of the concept of AM." They state - correctly in our judgment - that "it might be disputable to jump to the conclusion that: 'investments in public health infrastructure and increasing access to health services in megacities-both in China and worldwide-can produce significant mortality declines." Likewise, Cheng ${ }^{3}$ notes that our hypothesis is "data compatible... and plausible" but that we "cannot rule out alternative, plausible hypotheses on other factors that may have driven the decline in AM in Shanghai." Finally, Yip and $\mathrm{Chen}^{6}$ criticize us for not producing empirical data to "demonstrate a direct link for (the) causal relationship" between the decline in AM and improvement in Shanghai's healthcare system. In fact, we do provide some evidence of substantial investments in the healthcare system and Fabre's ${ }^{10}$ commentary highlights the emerging role of healthcare investments as part of China's new growth regime.

We are surprised by these reactions because they misconstrue the purpose of our analysis. We did not seek to validate the AM indicator. There is an extensive literature on this score including a recent analysis by Heijink and colleagues ${ }^{11}$ that finds a statistically significant relationship between healthcare spending and AM. All indicators are subject to limitations and caveats that must be examined before making claims about causality. Indeed, that is why they are called "indicators." We relied on the indicator, AM, because there is strong evidence in the literature we cited that this indicator tells us something important about one dimension of healthcare system performance. To suggest, as our critics have, that there are other important factors, aside from access to effective

Full list of authors' affiliations is available at the end of the article. 
healthcare, that have contributed to health improvements in Shanghai is indisputable. To imply that we should have disentangled the relative importance of these factors in the case of Shanghai simply because we relied on the AM indicator is unreasonable. It is, as if we relied on life expectancy at birth or infant mortality to compare levels of well-being and were told that there are many other factors to consider before making causal claims! For all those who recognize the overwhelming importance of social determinants of health $(\mathrm{SDH})$ in explaining population health status, we share your view. But for those who refuse to recognize that the AM indicator has something to add in assessing an important dimension of healthcare system performance, we recommend that you read Allin and Grignon's ${ }^{12}$ incisive defense of the indicator.

Needless to say, we recognize that the use of comparative rates of AM to estimate gains in health from improved health system performance is limited by lack of available data to assess more directly health system performance (eg, hospital readmission rates or admission rates for ambulatory care sensitive conditions or use of recommended primary and secondary clinical and preventive services. Similarly, without the collection of small-area socio-demographic measures, the influence of such factors as poverty cannot be determined. In our comparisons of world cities ${ }^{2}$, we have emphasized the extent to which New York City reflects national policy in the United States of not sufficiently (in our judgment) reducing inequities in the SDH, but also because the United States - even after passage of the Affordable Care Act (ACA) - has not come close to eliminating financial barriers in access to healthcare. Most other studies of these problems either emphasize the decisive impact of SDH or the importance of healthcare access. We have chosen a middle ground in which we pay attention to the consequences of poor social conditions, as well as access barriers to healthcare services that can prevent disease, improve health, reduce avoidable hospitalizations, and ultimately reduce premature mortality amenable to healthcare. ${ }^{13}$ It is for these reasons that we rely on the AM indicator, among others, to provide preliminary assessments of urban healthcare systems.

Our purpose in collaborating with colleagues in Shanghai was to show how analysis of available data on one important indicator of healthcare system performance can be used to provide a comparative perspective on achievements to date and to stimulate discussion of next steps in promoting useful research on how Shanghai's healthcare system can continue to improve. At the end of the day, we are convinced that it is not sufficient simply to state that health reforms have improved the healthcare system. ${ }^{14}$ Saying so does not make it so! Also, as we note in our paper and as all of the commentaries have reiterated, without data on the health status of Shanghai's migrant population, it is impossible to make legitimate claims about Shanghai's population health and its healthcare system. Finally, it will be important to conduct neighborhoodlevel analyses of population health and access to healthcare services to advance our capacity to assess the performance of Shanghai's healthcare system and make more meaningful comparisons with other world cities.
Competing interests

Authors declare that they have no competing interests.

Authors' contributions

VGR drafted the article based on discussions with MKG and DW. All authors reviewed final version.

\section{Authors' affiliations}

${ }^{1}$ The Hasting Center, Garrison, NY, USA. ${ }^{2}$ Wagner School of Public Service, New York University, New York City, NY, USA. ${ }^{3}$ The International Longevity Center, Columbia University, New York City, NY, USA.

\section{References}

1. Gusmano MK, Rodwin VG, Wang C, Weisz D, Lou L, Hua F. Shanghai Rising: health improvements as measured by avoidable mortality since 2000. Int J Health Policy Manag. 2015;4(1):7-12. doi:10.15171/ijhpm.2015.07

2. Rodwin VG, Weisz D. Health Care in World Cities: New York, London, Paris, Tokyo. Baltimore, MD: Johns Hopkins University Press, 2010.

3. Cheng TM. Shanghai's track record in population health status: what can explain it? Comment on "Shanghai rising: health improvements as measured by avoidable mortality since 2000". Int J Health Policy Manag. 2015;4:forthcoming. doi:10.15171/ ijhpm.2015.117

4. Marmor T, Freeman R, Okma K. Comparative Perspectives and Policy Learning in the World of Health Care. Journal of Comparative Policy Analysis: Research and Practice. 2005;7(4):331-348. doi:10.1080/13876980500319253

5. Ren Y. Health improvements for a healthy shanghai rising: Comment on "Shanghai Rising: health improvements as measured by avoidable mortality since 2000". Int J Health Policy Manag. 2015;4(3):189-190. doi:10.15171/ijhpm.2015.33

6. Yip $P$, Chen $M$. What really matters: living longer or living healthier? Comment on "Shanghai rising: health improvements as measured by avoidable mortality since 2000". Int J Health Policy Manag. 2015;4(7):487-489. doi:10.15171/ijhpm.2015.87

7. Rodwin VG. Gusmano MK. The world cities project: rationale and design for comparison of megacity health systems. J Urban Health. 2002:79(4):445-463.

8. World City Project Publications. http://wagner.nyu.edu/faculty/ vrodwinWCP

9. Yan F, Zhang J. Untimely applause was a distraction: Comment on "Shanghai rising: health improvements as measured by avoidable mortality since 2000". Int J Health Policy Manag 2015;4(6):403-405. doi:10.15171/ijhpm.2015.64

10. Fabre G. The Chinese healthcare challenge: Comment on "Shanghai rising: avoidable mortality as measured by avoidable mortality since 2000". Int J Health Policy Manag. 2015;4(3):195197. doi:10.15171/ijhpm.2015.36

11. Heijink R. Koolman X. Westert G. Spending more money, saving more lives? The relationship between avoidable mortality and healthcare spending in 14 countries. Eur $J$ Health Econ. 2013;14:527-538.

12. Allin S, Grignon M. Examining the role of amenable mortality as an indicator of health system effectiveness. Healthcare Policy. 2014;9(3)12-18. doi:10.12927/hcpol.2014.23733

13. Gusmano MK, Rodwin VG, Weisz D. Using comparative analysis to address health system caricatures. Int J Health Serv. 2014;44(3)553-565. doi:10.2190/hs.44.3.g

14. Cheng TM. Explaining Shanghai's health care reforms, successes and challenges. Health Aff (Millwood). 2013;32(12):2199-2204. doi:10.1377/hlthaff.2013.1136 